\title{
Chest computed tomography: a validated surrogate endpoint of cystic fibrosis lung disease?
}

\author{
Martine Loeve ${ }^{1,2}$, Gabriel P. Krestin², Margaret Rosenfeld ${ }^{3}$, Marleen de Bruijne ${ }^{4,5}$, \\ Stephen M. Stick ${ }^{6}$ and Harm A. Tiddens $s^{1,2}$
}

\author{
Affiliations: \\ 1Dept of Paediatric Pulmonology and Allergology, Erasmus Medical Center-Sophia Children's Hospital, \\ Rotterdam, \\ ${ }^{2}$ Dept of Radiology, Erasmus Medical Center, Rotterdam, and \\ ${ }^{4}$ Biomedical Imaging group Rotterdam, Depts of Radiology \& Medical informatics, Erasmus MC, Rotterdam, \\ The Netherlands. \\ ${ }^{3}$ Division of Pulmonary Medicine, Dept of Paediatrics, University of Washington School of Medicine, Seattle, \\ WA, USA. \\ ${ }^{5}$ Dept of Computer Science, University of Copenhagen, Denmark \\ ${ }^{6}$ Division of Pulmonary Medicine, Princess Margaret Hospital for Children, Perth, Australia.
}

\section{Correspondence:}

H.A. Tiddens, Dr. Molewaterplein 60, 3015 GJ Rotterdam, The Netherlands.

E-mail: h.tiddensderasmusmc.nl

ABSTRACT Clinical trials for the treatment of cystic fibrosis (CF) lung disease are important to test and optimise new therapeutic interventions. To evaluate the effect of these interventions, sensitive and accurate outcome measures are needed. The most commonly used endpoints are spirometric variables such as the forced expiratory volume in $1 \mathrm{~s}$ and respiratory tract exacerbations. Unfortunately, these endpoints are relatively insensitive for monitoring progression of CF lung disease, and thus require a large number of patients when used in clinical studies. In addition, these endpoints are not suitable to study CF lung disease in young children. Chest computed tomography (CT) holds great promise for use as a sensitive surrogate endpoint in CF. A large body of evidence has been produced to validate the use of chest CT as a primary endpoint to study CF lung disease. However, before chest CT can be used in clinical trials, it has to be recognised as a validated surrogate endpoint by regulatory agencies. The aim of this review is to summarise what is currently known about the use of chest CT as surrogate endpoint in clinical trials in CF.

@ERSpublications

Evaluation of the use of chest CT as a sensitive surrogate endpoint in clinical trials in CF http://ow.ly/mEJE7

\section{Received: March 272012 | Accepted after revision: Nov 282012 | First published online: Dec 202012}

Support statement: This study was supported by grants from the Sophia CF research fund, the Dutch Cystic Fibrosis Foundation (NCFS), and the Italian CF Fund (IERFC). None of the sponsors was involved in the study design, data collection, analysis, interpretation of the data, writing of the report or in the decision to submit the paper for publication.

Conflict of interest: Disclosures can be found alongside the online version of this article at www.erj.ersjournals.com

Copyright @ERS 2013 


\section{Introduction}

Cystic fibrosis (CF) lung disease is the primary cause of death in most CF patients. Thanks to improved treatment, median survival currently approaches 38 years [1]. New therapeutic agents, such as inhaled antibiotics and disease modifying agents, are in development to further improve treatment [2-4]. To test the effectiveness and safety of these drugs in randomised clinical trials, sensitive and accurate outcome measures are needed [5]. Before an outcome measure can be used as a primary endpoint in a clinical study, it has to meet stringent requirements. First, it must reflect the presence and severity of the disease. Secondly, it has to reflect a clinically meaningful improvement in disease severity when the disease is treated effectively. Thirdly, the measurement must be reproducible. Fourthly, changes in the endpoint should closely match changes in the true outcome [6]. Two types of endpoints can be distinguished: clinical or "true" endpoints, and surrogate endpoints. The US Food and Drug Administration (FDA) defines clinical endpoints as direct measures of how a patient feels, functions, or survives, and are thus expected to predict the effect of a therapy. The FDA defines surrogate endpoints as laboratory measurements or physical signs that can be used as a substitute for a clinical endpoint [7]. Surrogate endpoints are generally used when the clinical benefit may not be detectable in trials of reasonable cost, duration or size [8]. FDA regulations state that a surrogate endpoint is considered to be "reasonably likely to predict clinical benefit and, therefore, useable for drug approval if there is evidence based on epidemiologic, therapeutic, pathophysiologic, or other data supporting the association of the surrogate with the clinical benefit" [8].

In CF, two clinical endpoints are currently used: respiratory tract exacerbation rate (RTE-R) and quality of life (QoL) [9]. RTE-R is an important endpoint that has been shown to increase with age and more severe lung function impairment [10]. In addition, there is a clear association between RTE-R and survival in CF [11]. Unfortunately, the use of RTE-R has disadvantages. It is a relatively insensitive endpoint, especially in (young) children with early lung disease, who generally experience less frequent exacerbations compared with older children. As a result, RTE-R requires a large sample size when used in clinical studies [12]. Furthermore, there is no universal consensus regarding the definition of an RTE [13].

QoL is another important clinical endpoint. The FDA accepts patient-reported outcome measures as primary or secondary endpoints if they are appropriate for the disease, product and indication [14]. QoL has the advantage of measuring directly how a patient reports to feel or function. The two most commonly used CF-specific QoL questionnaires (the CF QoL questionnaire and the revised CF QoL questionnaire $(C F Q-R))$ are well validated with demonstrated reliability, validity and sensitivity $[15,16]$. The CFQ-R has been used as a primary endpoint in a study evaluating the effectiveness of an inhaled antibiotic against Pseudomonas aeruginosa [17]. However, to date, there is still a lack of systematically validated QoL questionnaires in young children. In addition, further work is needed to standardise the use of QoL instruments in clinical research. Furthermore, the development of new QoL instruments that are sensitive to smaller and earlier changes in symptoms is needed, as improvements in overall health and lung function are seen in successive CF birth cohorts [14].

The most commonly used surrogate endpoint in $\mathrm{CF}$ is the forced expiratory volume in $1 \mathrm{~s} \mathrm{(FEV1).} \mathrm{FEV1} \mathrm{is}$ indirectly related to structural lung damage. When structural damage affects large lung volumes, low values are observed [18]. Thanks to improvements in CF therapy over the past decades, FEV1 has become relatively insensitive for monitoring CF lung disease progression. Currently, FEV1 is in the normal range for most patients until adolescence, and the average annual FEV1 decline is less than 1\% [19]. In addition, FEV1 is insensitive to detect early and localised structural changes [18], is difficult for young children to perform and is not suitable for infants and most pre-school children [9]. Thus, there is a need for new, more sensitive surrogate endpoints in CF that reflect mild lung disease and that can be used to assess lung disease in infants and young children.

A promising endpoint that has been extensively studied since the mid-1990s is chest computed tomography (CT) images of the lungs. Importantly, using chest CT, structural abnormalities can be easily observed. Various methods have been developed to quantify these structural abnormalities. In addition, a large number of studies have been carried out to validate chest CT as a surrogate endpoint. The aim of this review is to summarise what is currently known about the use of chest CT as a surrogate endpoint in clinical trials in CF. In addition, we will discuss its advantages and disadvantages, and the future work needed to further improve sensitivity and accuracy of chest CT for use in clinical trials.

\section{CF lung disease}

At birth, CF patients have macroscopically normal lungs [20]. The structural changes related to CF, however, occur early. Pathology studies have shown the presence of structural abnormalities such as bronchiectasis and mucus impaction even in infants aged 0-4 months with CF [21]. In addition, all children 
in this age group were found to have evidence of bronchial wall inflammation. Airway wall thickening, a frequently observed abnormality in CF, has been correlated with airway inflammation [22, 23$]$. In other lung diseases, such as chronic obstructive pulmonary disease (COPD) and asthma, the severity of airflow obstruction is proportional to the severity of airway wall thickening $[22,24]$. Similar correlations have been reported in CF. Furthermore, a threefold increase in airway wall thickening was found in specimens from CF patients compared with specimens from COPD patients [23]. In addition, destruction of the epithelial layer and a substantial loss of cartilage have been described in CF $[25,26]$. All these factors are likely to contribute to the airflow obstruction that is present in CF [23]. To monitor CF-related lung changes in vivo, CT scanning can be used, as it was shown to correlate well to pathological findings, such as bronchiectasis [27].

\section{Image analysis}

An important condition for the use and validation of chest CT as surrogate endpoint is that the structural abnormalities can be quantified in a reproducible manner. This can be done using manual (semi-) quantitative scoring systems and/or automated systems.

\section{Manual scoring systems}

For CT scoring, a range of systems is available with proven reproducibility. In a comparative study, withinand between-reader reproducibility for five scoring systems was found to be good with intraclass correlation coefficients of $\geqslant 0.74$ [28]. These semi-quantitative scoring systems evaluate structural abnormalities by assigning scores ranging from 0 to 3 for severity and/or extent. These systems were mainly developed to quantify abnormalities in mild-to-moderate CF lung disease. For CF patients with severe advanced lung disease (SALD), a dedicated SALD scoring system was developed [29]. This system uses a digital grid to annotate all lung tissue into 4 components: 1) infection/inflammation (including several abnormalities, from which bronchiectasis is the most important one); 2) trapped air/hypoperfusion; 3) bulla/cysts; and 4) normal/hyperperfusion. The SALD system has been shown to correlate with the Brody-II system developed for mild disease, with good reproducibility between and within observers [29].

\section{Automated systems}

Ideally, automated systems should be used for CT quantification, as they allow more rapid analyses with near perfect intratest reproducibility. Various semi-automated [30-35] and fully automated [36-43] systems have been developed. The semi-automated systems have been shown to be capable of measuring airway wall thickness, bronchial diameter [30, 31] and trapped air [32, 33]. These systems have been validated in $\mathrm{CF}$, and could differentiate patients from controls [31-33]. Furthermore, the parameters have been correlated with visual scores from radiologists [30, 31] and pulmonary function tests (PFTs) [30-33]. For longitudinal follow up, progression of trapped air has been tracked. In a study by LoEve et al. [34], two routine expiratory CTs over 2 years were matched, and the proportion of stable, disappeared and new trapped air was measured. With this novel approach, trapped air was found to have a stable component. Quantitative trapped air measurements have been used in intervention trials [35]. In a 1-year randomised placebo-controlled trial with dornase $\alpha, 25$ children with mild CF were enrolled. The results showed that quantitative trapped air measures could discriminate differences in treatment effects in children with mild $\mathrm{CF}$ at one of the two study visits. The disadvantages of semi-automated systems are that they require user input to mark the trachea [31], airway wall and vessels [30] or thresholds for trapped air [32-34]. This is in contrast to fully automated systems that require no user input. Currently available automated systems are able to quantify the bronchial tree [40-42] and measure airway wall thickness and bronchial diameter $[36,38,39,43-44]$. In addition, a system was proposed for matching and labelling of airway trees to allow within- and between-patient comparisons [41]. These automated systems were validated using CT images from lung cancer screening trials [38], smokers [39], healthy subjects [39, 42], a random set of cases [37, 44], asthma patients [43] or phantoms [39, 40, 42]. Measurements correlated well with PFTs [38] and manual expert readings [36, 37, 41]. Unfortunately, validation in CF is lacking in all but one study [40]. In addition, there are still substantial technical issues to be resolved. For example, the influence on the measurements by factors such as scan parameters, inflation level, effect of visual input on reproducibility for the semi-automated systems and clinical validity of the measurements [45]. Thus, automated systems are promising but further development is needed before these systems can be used in clinical trials.

\section{Presence and severity of disease}

The first validation requirement for an endpoint is that it must reflect the presence and severity of the disease. Currently, chest CT is considered the gold standard for detection of bronchiectasis [46]. An important advantage of CT is that it easily allows recognition of the structural lung abnormalities characteristic for CF, which are not present in normal subjects. Furthermore, CT can detect abnormalities early. In cohort studies in children with CF diagnosed through newborn screening structural abnormalities, 
such as bronchiectasis, trapped air and mucus impaction, could be observed even in asymptomatic infants [47-50]. In addition, bronchiectasis in infants and older children was shown to be progressive [47, 51-53]. In children with CF diagnosed through newborn screening, bronchiectasis was present on chest CT in around $10 \%$ in the first year of life. By the age of five years over $50 \%$ of children had bronchiectasis $[47,54]$. Trapped air on expiratory CT was observed in two-thirds of children with CF in the first year of life and continued to persist in around two-thirds in the first 5 years of life [47, 53-54]. Furthermore, CT proved to be more sensitive in detecting and monitoring bronchiectasis and other structural abnormalities than PFTrelated parameters (fig. 1) [51-52]. Finally, bronchiectasis and trapped air are the most important components of end stage CF lung disease [29]. Other structural abnormalities that can be observed on chest CT in CF are airway wall thickening, consolidations and bulla/cysts. An important advantage of CT over PFTs is that structural lung abnormalities can be differentiated in the above described components.

To summarise, there is a large body of data supporting the concept that CT is sensitive to detect the presence, and monitor the severity, of structural changes relevant for patients with CF starting in infancy into adulthood.

\section{Response to treatment}

The second validation requirement for an endpoint is that it has to show improvement upon successful treatment of the disease. CT scores have been shown to improve with antibiotic treatment for an RTE. SHAH et al. [55] studied 27 adult CF patients and showed that CT scores for airway wall thickening, mucus and air fluid levels in bronchiectasis and centrilobular nodules improved in 2 out of 19 (11\%), 6 out of 18 (33\%), 2 out of $2(100 \%)$ and 4 out of $11(36 \%)$ of patients, respectively, in response to treatment. Similar findings were reported for paediatric patients. RoBINSON et al. [56] studied 17 paediatric and adult CF patients (mean age 17.2 years), and showed that CT total and mucus score was significantly reduced after treatment for an RTE. BRODY et al. [57] studied eight paediatric CF patients (mean age 12.7 years) experiencing a total of 15 RTEs, and showed that CT airway wall thickening and mucus scores significantly improved after treatment. Even in very young children, these improvements were observed. DAvis et al. [58] studied 13 young children with CF (mean age 17 months) and showed that CT total, combined bronchiectasisbronchial dilatation and hyperinflation scores significantly reduced after treatment.

CT score improvement after treatment with dornase $\alpha$ and tobramycin solution for inhalation is less well established. ROBINSON et al. [35] studied 25 children with CF and showed that quantitative trapped air score and mean total mucus score decreased significantly in the dornase $\alpha$ group compared with placebo. However, these effects were only significant at one of the two study visits [35]. Using a different scoring system, these CT scores could discriminate between treatment and placebo group at one of the two study visits but only when combined with PFTs as a composite score [59]. Modified scores were also used in other studies. Studying dornase $\alpha$, NASR et al. [60] reported significant increases in CT scores in the treatment group versus placebo. However, this was only present when CT scores were calculated as a "gain score" [60]. Studying tobramycin solution for inhalation, they reported a trend towards improvement after one cycle of treatment using a modified total CT score [61]. After three treatment cycles, they found a significant decrease in airway wall thickening score in the treatment group versus placebo [62]. Bronchiectasis is an

FIGURE 1 Image showing the dissociation between lung function and lung structure assessed by computed tomography (CT). Shown is a slice of a routine CT scan of a cystic fibrosis patient, performed during an annual check-up when clinically stable. This slice clearly shows bronchiectasis and bronchial wall thickening (white arrows), and mucus plugging (black arrow). However, spirometry parameters at the time of the CT were in the normal ranges, with a forced expiratory volume in $1 \mathrm{~s}$ of $98 \%$ predicted.

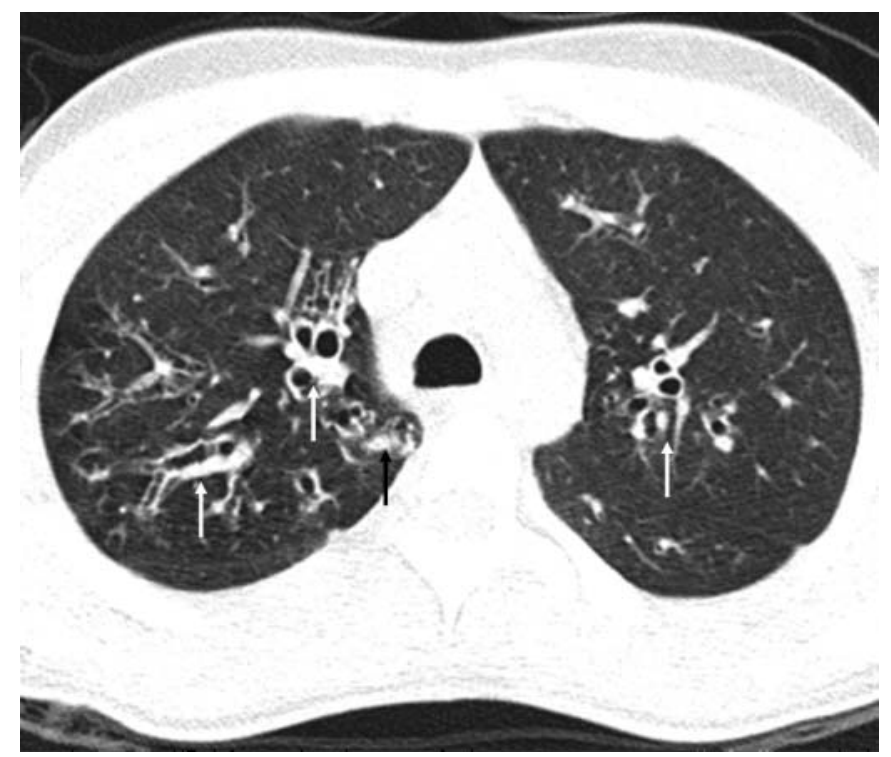


important outcome parameter. Since bronchiectasis is defined as an irreversible structural change, intervention studies can investigate whether the development of bronchiectasis can be prevented or whether its progression can be slowed down. All the aforementioned intervention studies included bronchiectasis as an outcome measure. Surprisingly, despite the irreversible nature of bronchiectasis, decreased bronchiectasis scores were observed in the intervention group in all but one study [35, 59-62]. However, whether the observed reductions in bronchiectasis score were statistically significant was not stated. Most likely, these reductions can be explained by the CT protocols used. Most of the aforementioned studies used limited slice protocols. It is well recognised that the variability of these protocols to detect bronchiectasis is higher and the sensitivity lower relative to volumetric CT protocols [63].

Thus, while other CT scores can improve with treatment, the effect of treatment on bronchiectasis needs to be further studied. Until the efficacy of antibiotics, mucolytics or disease modifiers on the development of bronchiectasis has been reported, this requirement for bronchiectasis score has not yet been completely met.

\section{Standardisation}

The third validation requirement for endpoints is reproducibility of the measurement. For CT, it is important to standardise the scanning protocol to optimise reproducibility. The most important variable that requires standardisation is the lung volume during scanning. Traditionally, in patients aged 5 years and above voluntary breath holding is used during scanning. Patients are instructed by the CT technician or a recorded voice to perform a maximal inhalation manoeuvre and then hold their breath during scanning to obtain end-inspiratory images. Next, patients are asked to maximally exhale and hold their breath to obtain end-expiratory images. With this technique, however, one cannot assess whether a patient was able to follow the instruction correctly and hold his or her breath at the maximum in- and exhalation level. For many patients, and specifically for children, these manoeuvres are difficult to perform. In a paediatric study (mean age 12 years), voluntary breath hold scan volumes were compared with plethysmographic lung volume measurements prior to scanning. Average inspiratory volume was acceptable at $77 \%$ of total lung capacity (TLC), but ranged $55-106 \%$. Average expiratory volume was $86 \%$ of functional residual capacity (FRC) and $140 \%$ of residual volume (RV), suggesting that expiratory manoeuvres are even more difficult for children [64]. Thus, there is a need for better standardisation of breath holding during CT scanning. This can be obtained by using a spirometer combined with instructions from a lung function technician. Prior to scanning, the patient practices the breathing manoeuvres with the spirometer coached by the technician. Next, the patient performs these manoeuvres during scanning, again coached by the lung function technician [65]. Scanning will commence when the required volumes are obtained by the patient. The aim of this procedure is to obtain an inflation level $\sim 95 \%$ of slow vital capacity (SVC), and an expiration level $\sim 5-12 \%$ of SVC $[48,66]$. Recently, the volume-monitored technique has been described. This method combines coaching with a portable spirometer that generates respiratory tracings to aid the patient in achieving targeted lung volumes [67]. With this method, patients as young as 4 years old were able to achieve reproducible images at $95 \%$ of full inflation and at $77 \%$ of vital capacity for the expiratory images.

In very young children, lung volume standardisation requires a different approach. For these children, a non-invasive pressure-controlled ventilation (PCV) technique under general anaesthesia or sedation has been introduced [48]. This technique requires no intubation and starts off by hyperventilating the child by giving a series of augmented breaths using positive pressure applied via a facemask to induce a physiological respiratory pause. During this pause, the lungs are imaged at full inflation by maintaining a positive facemask pressure $\left(25-30 \mathrm{cmH}_{2} \mathrm{O}\right)$ for inspiratory images, and resting end-exhalation by applying no mask pressure for expiratory images $[48,66]$. Both spirometer and PCV techniques have been shown to be highly reproducible $[66,68]$.

Volume control is particularly important for assessment of bronchiectasis and trapped air. In a paediatric study, bronchiectasis was identified on 30\% of images obtained at end-inspiration using PCV techniques compared with $6 \%$ of images obtained during quiet breathing. Trapped air was seen in $45 \%$ of images obtained at endexpiration compared with $19 \%$ of images obtained during quiet breathing [69]. Thus, for standardisation of chest CT, volume control is an important condition for both inspiratory and expiratory scans.

\section{Correlation with true endpoints}

The fourth validation requirement for endpoints is that changes in the endpoint should closely match changes in the true outcome. The first argument showing that CT meets this criterion is that higher CT scores have been correlated with characteristics associated with more severe disease. For example, higher CT scores were reported for patients with pancreatic insufficiency compared with patients with sufficient pancreatic function [70]. In addition, CT scores strongly correlated with Pseudomonas aeruginosa 
acquisition, a well-established risk factor for progressive CF lung disease [71-73]. The second argument that CT meets this criterion, is that CT scores have been linked to the true endpoints RTE-R, QoL, and survival.

RTE-R

Two studies have shown a correlation between severity of structural changes on CT and RTE-R [74-75]. The first study described 61 subjects from the Pulmozyme Early Intervention Trial, including 6-10-year-old children with well-preserved lung function (forced vital capacity $\geqslant 85 \%$ ) [74]. CTs and PFTs were performed at the beginning and end of the 2-year trial during which RTE-R was recorded. Nine out of 61 subjects experienced a total of 22 RTEs. PFTs and CT scores at baseline correlated significantly with RTE-R $\left(\mathrm{r}_{\mathrm{s}}-0.40-0.30\right)$, although none of these variables predicted RTEs with high accuracy [74]. The second study was performed in an unselected cohort of 115 children and adolescents with CF [75]. In this retrospective study, routine chest CTs and PFTs performed during annual check-up were collected with 2 years' follow up in which RTE-R was recorded. 51 subjects experienced a total of 148 RTEs. Bronchiectasis was found to be the strongest predictor of RTEs of all CT parameters, and added significantly to the predictive value of FEV1.

QoL

A recent study has shown an association between bronchiectasis and impaired QoL [76]. In this crosssectional study, the effect of CT scores on QoL was assessed using routine CTs and CFQ-Rs from 72 children and adolescents with CF. CTs were scored using a modified Brody-II scoring system. Significant correlations between the respiratory domain and bronchiectasis and trapped air scores were found $\left(\mathrm{r}_{\mathrm{s}}-0.39\right.$ and -0.30 , respectively).

\section{Survival}

Recently, a correlation between CT and survival has been established. In a multi-centre study including 366 CF patients awaiting lung transplantation, routine CT scans acquired at the time of screening were scored with the (semi-automated) SALD scoring system [29]. SALD infection/inflammation score (including bronchiectasis) was significantly correlated with waiting list survival, and added to the predictive value of the lung allocation score [77]. The correlation with survival is an important addition to the portfolio of CT as an outcome measure.

\section{Correlation with other surrogate endpoints}

In addition to correlations with true endpoints, CT parameters have also been linked to the following frequently used surrogate endpoints.

\section{Spirometry parameters}

Several studies have shown a correlation between CT and spirometry parameters such as FEV1, currently the most extensively validated surrogate endpoint in $\mathrm{CF}$. FEV 1 is indirectly related to structural lung damage and has been shown to correlate with survival [11, 78-88]. From these studies we learned that CT scores are more sensitive than PFTs in detecting and monitoring onset and progression of CF lung disease [51-52]. Comparing the validation status of FEV 1 to $\mathrm{CT}$, it can be concluded that most of the validation steps for CT have been addressed (table 1).

\begin{tabular}{|c|c|c|}
\hline Parameter & FEV1 & Ст \\
\hline Presence and severity of CF lung disease & {$[18,19,83,86,89-96]$} & {$[29,46,47,49,50,52,91,97,98]$} \\
\hline Response to treatment in CF & {$[35,56,99-120]$} & {$[35,55-60,62]$} \\
\hline Reproducibility of the measurement & {$[121-129]$} & {$[28,29]$} \\
\hline \multicolumn{3}{|l|}{ Link to true outcomes in $\mathrm{CF}$} \\
\hline Respiratory tract exacerbations & {$[10,74,75,84,92,99,130]$} & {$[74,75]$} \\
\hline Survival & {$[11,78-88]$} & {$[77]$} \\
\hline Quality of life & [131-143] & {$[76,144]$} \\
\hline
\end{tabular}

Table shows cited references for each endpoint. FEV1: forced expiratory volume in $1 \mathrm{~s}$; CT: computed tomography; CF: cystic fibrosis. 


\section{Lung clearance index}

CT scores have also been shown to correlate with lung clearance index (LCI), a promising early marker derived from multiple breath washout [145-147]. The LCI has been shown to be more sensitive in the detection of lung disease than spirometry [145, 148-151]. In addition, LCI is reproducible [148, 151], and can be performed in infants [152]. Its narrow range in normal subjects makes it a suitable measure for long term follow up [145, 148-151]. A normal LCI has even been suggested to exclude structural changes on CT $[145,146]$, a finding that was not supported by another study [147]. However, its sensitivity to monitor progression of advanced lung disease has not yet been investigated. In addition, LCI has not been well validated against other true and surrogate endpoints.

\section{Inflammatory parameters}

The severity of structural changes on CT has been shown to correlate with inflammatory parameters in bronchoalveolar lavage (BAL). In a study in 17 children under the age of 4 years, regional distribution of airway disease was assessed using CT scans, and abnormalities were correlated to markers of lower airway inflammation provided by BAL. They found that in the lobe with greatest disease, as indicated by CT, inflammatory markers were higher than in lobes with least disease [58]. These findings have been confirmed in two other studies in infants with CF identified by newborn screening who underwent BAL and CT scanning as part of an early surveillance program $[47,50]$.

Thus, CT has been shown to be clearly linked to a number of clinically meaningful outcome measures. These correlations are essential in the validation of CT as surrogate endpoint in CF.

\section{Advantages of using CT in clinical trials}

In the previous paragraphs, we have shown that CT meets many of the requirements for surrogate endpoints. CT is able to detect structural changes relevant for CF patients, abnormalities on CT can be reproducibly quantified, and standard CT scanning procedures have been defined. CT scores can improve with treatment, although this requirement has not been completely met for bronchiectasis. In addition, CT parameters have been linked to other clinically meaningful outcome measures. Thus, CT can be considered quite validated as a surrogate endpoint for CF-related lung disease. Using CT as surrogate endpoint has the following advantages. First, CT can easily be performed in most CF centres, as virtually all centres are equipped with a CT scanner. Secondly, CT can be performed across all age ranges, including infants. Thirdly, CT is the most sensitive tool to detect early and regional disease. Compared with spirometry parameters, CT is more sensitive to detect and monitor disease progression [51-52, 98]. It has been estimated that the better sensitivity of CT relative to PFTs can substantially reduce sample size in clinical studies $[5,70]$. This would increase the feasibility of running clinical trials in CF.

\section{Disadvantages of using CT in clinical trials}

Clearly, the use of chest CT in clinical trials has a number of disadvantages such as ionising radiation, the need for general anaesthesia in young children, costs and the relative complicated procedures needed for the pressure-controlled volume scanning.

\section{Radiation}

CT exposes patients to ionising radiation, which increases one's natural life-long risk of cancer. Children are particularly at risk, as tissues and organs are growing and developing and are therefore more sensitive to radiation. In addition, children have a longer life expectancy and therefore more time to manifest the oncogenic effects. At high exposures, the risk of cancer increases linearly with increasing dose. The relationship between radiation exposure and cancer risk from low-dose radiation (such as CT scanning) is less clear. In a study using a computational model, bi-annual chest CTs (mean dose $1 \mathrm{mSv}$ ) were shown to carry a low risk of radiation-induced mortality, with reported cumulative cancer mortalities of $1 \%$ at age 40 years and $6 \%$ at age 65 years [153]. In addition, the association between radiation dose from CT scans and leukaemia and brain tumours has been reported recently, with risk estimates in even a lower range than those previously stated [154]. However, with increasing overall survival, life-long radiation exposure also increases and thus the risk of radiation-induced cancer and mortality can become more meaningful. Therefore, protocols should aim to limit radiation to the absolute minimum needed to acquire images of sufficient quality. Six-slice protocols with a mean dose of $0.19 \mathrm{mSv}$ have been described and were found to be appropriate for evaluating bronchiectasis in paediatric patients [155]. However, limited-slice CT reduces the ability to identify specific areas and their interval change, thus decreasing its ability to detect a therapeutic effect. Volumetric CT scanning will improve the ability to identify and compare specific structures on serial CTs. Currently, we can acquire volumetric CTs (inspiratory plus expiratory images) of sufficient quality for assessment of structural changes with a mean total effective dose near $1 \mathrm{mSv}$ [64]. This 
is comparable to one-third of the annual US background radiation [156]. These doses are likely to be further reduced in the near future [64]. Keeping the risk-benefit ratio of clinical trials in mind, CT should be considered as surrogate endpoint in studies aiming to slow the progression of CF lung disease.

\section{Sedation/anaesthesia}

A second disadvantage is that CT scanning in infants and young children requires sedation (i.e. with chloral hydrate) or general anaesthesia. These agents are very effective, with reported sedation failure rates of less than $1 \%$ using chloral hydrate [157]. In addition, they have a good safety profile. In two large retrospective studies reviewing records of children who underwent sedation for imaging studies, adverse events were reported in $0.85 \%$ and $0.42 \%$ of patients, respectively $[157,158]$. Recent animal studies and experimental data have raised concerns about the potential neurotoxicity of sedation and anaesthetic agents and their effect on the developing brain $[159,160]$. To date however, there is still insufficient evidence to determine whether anaesthetics are harmful to the developing human brain [161]. Thus, the risks of sedation are low, but not zero. To minimise these risks, patients should be carefully selected and monitored [162] and sedated using the most suitable technique [163]. The advantage of sedation/anaesthesia is that lung volumes can be standardised. Sedation/anaesthesia can be avoided using recently developed ultra-fast CT scanners. A comparative study showed that excellent image quality could be achieved in infants using a second generation $(2 \times 64$ detector rows $)$ dual source CT without sedation with a radiation dose similar to that of conventional scanners [164]. However, CTs acquired during free breathing are usually taken at a volume level near FRC. Whether the sensitivity and accuracy of these scans to detect structural abnormalities is comparable to that of volume-controlled inspiratory and expiratory scans has not yet been investigated.

\section{Costs}

A third disadvantage of CT is the high costs. The use of CT as surrogate endpoint will increase the costs of an intervention study. However, CT likely reduces the required sample size, which ultimately reduces the total cost of a trial. To the best of our knowledge, the costs and cost-effectiveness of CT in CF clinical trials has never been investigated. More research is needed to investigate this further.

\section{Volume control}

A fourth disadvantage is that CT protocols including PCV techniques or use of a spirometer are relatively complicated and require a trained team. Children aged $\geqslant 5$ years need to be trained and coached by lung function technicians before and during scanning. In addition, a spirometer is required for use in the CT room. Procedures involving children aged $<5$ years require personnel experienced in paediatric sedation/ anaesthesia and the PCV procedure. Furthermore, adequate time slots must be available to execute the protocol. This may limit its feasibility in multicentre trials.

\section{Chest CT for clinical use}

Over the past decade, more and more centres have adopted the routine of performing a chest CT every other year at the time of the annual check-up in combination with PFTs [51]. A few centres perform a CT scan every third year [52]. This strategy is considered useful to determine the long-term efficacy of maintenance therapy. The most important reason for this routine is that chest CT is our current gold standard to detect bronchiectasis [46]. As discussed, chest CT provides a more sensitive and accurate estimate of the severity and progression of CF lung disease compared to PFTs [51-52]. In 50\% of patients, discordance is observed between PFTs and chest CT data. Several studies have shown that, despite stable spirometry parameters, CT scores show an annual progression ranging $0.8-2.2 \%$ for total CT score, $1-1.7 \%$ for bronchiectasis, $1-1.4 \%$ airway for wall thickening, $0.4-0.65 \%$ for mucus plugging and $1-7 \%$ for trapped air $[51,52,74,75]$.

Clearly, a multitude of modalities including PFTs are used to monitor CF lung disease and each of these modalities contribute to clinical decision making. For this reason, it is nearly impossible to determine the impact of chest CT to the prognosis contribution. A question often raised is in how far CT affects clinical management. In cases for which progression of bronchiectasis on CT is observed, adjustment to the treatment for the 2 years following the CT study should be made with the aim of stopping further progression. For clinical management, the CT interval of 2 years is in general too long for the timely identification of fast progressing patients. Due to the radiation related to chest CT, this modality should not be routinely used to monitor CF lung disease over short time intervals, such as for the identification of exacerbations. To monitor the course of CF lung disease during the time interval between bi-annual chest CTs, lung function measurements like spirometry and LCI will continue to play an important role. When a more intensive monitoring protocol of lung structure is needed for a high-risk patient, one can consider alternating routine bi-annual chest $\mathrm{CT}$ with bi-annual chest magnetic resonance imaging or with an 
ultra-low dose expiratory CT scan to reduce cumulative radiation dose [64]. Further reductions in radiation dose in the near future will increase our options.

\section{Future research}

In this review, we have presented a large body of evidence supporting the use of chest CT as a surrogate endpoint in clinical studies. The following further improvements can be made to increase the accuracy and sensitivity of the technique.

\section{Calibration standardisation}

CT has already shown its feasibility in multi-centre trials [71, 74, 165]. These trials demonstrated the importance of defining a detailed protocol to improve compliance, and to document the ability of the centres to apply this protocol [166]. CT studies require well-defined standard operating procedures to guarantee optimal image resolution and the use of the correct reconstruction algorithms. Since CT technology is moving fast, the procedures will require frequent updating.

Image analysis

Standardisation is also important for CT quantification. Agreement on the most appropriate scoring systems for the different CF cohorts (infants, children and adults) is necessary, in addition to commercial availability. Currently available fully automated systems require validation in $\mathrm{CF}$, and their usefulness in longitudinal studies should be determined. Such systems can further improve the sensitivity and accuracy of chest CT as a surrogate endpoint.

\section{Further validation steps}

An important next step will be to study the efficacy of antibiotics, mucolytics or disease modifiers on the development of bronchiectasis to further validate the response to treatment. In addition, it will be necessary to demonstrate that the effect of an intervention on the CT score predicts the effect on true clinical endpoints, such as RTE-R and QoL. This validation is specific to the class of intervention, and the CT score studied. Different CT scores may capture different types of structural changes, and therefore may affect different primary endpoints [5]. Furthermore, it is important to establish the minimal clinically relevant changes for each CT score. Thus, more comparative studies are needed. More longitudinal studies will help to further establish the trends of the different CT features over time. This will help defining the required time span for intervention studies. Ideally, a profile should emerge defining the therapeutic indications and target populations where CT will be most useful and likely to show change.

\section{Summary}

In this review, we have shown that CT scores meet all critical requirements for surrogate endpoints. CT is able to detect structural changes highly relevant for CF patients using standardised image acquisition protocols, and the abnormalities detected using CT can be reproducibly quantified. Most CT scores can improve with treatment, although this needs to be further established for the bronchiectasis score. In addition, CT has been linked to a number of clinically meaningful outcome measures. CT can be performed across all age ranges, is the most sensitive tool to assess mild and regional disease, and likely reduces sample size requirements for clinical studies compared to other recognised endpoints. The disadvantage of chest CT in clinical studies however, includes the radiation exposure to the patient, the need for sedation in infants, high costs and the more complicated procedures when volume control is needed. Further innovations in image analysis techniques will help to further improve sensitivity and accuracy of chest CT to monitor CF lung disease. Remaining challenges in the use of CT as surrogate endpoint in clinical trials comprise standardisation of CT protocols, procedures and quantification, better assessment of accuracy, and reliability of CT scores, better understanding of the association of CT scores with clinical outcomes, together with assessment of its feasibility in multi-centre settings.

\section{References}

1 Cystic Fibrosis Foundation. Patient Registry 2011 Annual Data Report. Bethesda, Cystic Fibrosis Foundation, 2012. www.cff.org/UploadedFiles/research/ClinicalResearch/2011-Patient-Registry.pdf Date last accessed: July 2, 2013.

2 Bals R, Hubert D, Tummler B. Antibiotic treatment of CF lung disease: from bench to bedside. J Cyst Fibros 2011; 10: Suppl. 2, S146-S151.

3 Becq F, Mall MA, Sheppard DN, et al. Zegarra-Moran, Pharmacological therapy for cystic fibrosis: from bench to bedside. J Cyst Fibros 2011; 10: Suppl. 2, S129-S145.

4 Conese M, Ascenzioni F, Boyd AC, et al. Gene and cell therapy for cystic fibrosis: from bench to bedside. J Cyst Fibros 2011; 10: Suppl. 2, S114-S128.

5 Emond MJ. Statistical power, reproducibility, and accuracy considerations for computed tomography scores as an endpoint in cystic fibrosis clinical trials. Proc Am Thorac Soc 2007; vol 4: p. E1-E6. 
Temple RJ. A regulatory authority's opinion about surrogate endpoints. In: Nimmo WS, Tucker GT, eds. Clinical Measurement in Drug Evaluation. New York, J Wiley, 1995; pp. 3-22.

Temple R. Are surrogate markers adequate to assess cardiovascular disease drugs? Jama 1999; 282: 790-795.

Katz R. Biomarkers and surrogate markers: an FDA perspective. NeuroRx 2004; 1: 189-195.

Rosenfeld M. An overview of endpoints for cystic fibrosis clinical trials: one size does not fit all. Proc Am Thorac Soc 2007; 4: 299-301.

10 Goss CH, Burns JL. Exacerbations in cystic fibrosis. 1: Epidemiology and pathogenesis. Thorax 2007; 62: 360-367.

11 Liou TG, Adler FR, Fitzsimmons SC, et al. Predictive 5-year survivorship model of cystic fibrosis. Am J Epidemiol 2001; 153: 345-352.

12 Mayer-Hamblett N, Ramsey BW, Kronmal RA. Advancing outcome measures for the new era of drug development in cystic fibrosis. Proc Am Thorac Soc 2007; 4: 370-377.

13 Rosenfeld M, Emerson J, Williams-Warren J, et al. Defining a pulmonary exacerbation in cystic fibrosis. J Pediatr 2001; 139: 359-365.

14 Goss CH, Quittner AL. Patient-reported outcomes in cystic fibrosis. Proc Am Thorac Soc 2007; 4: 378-386.

15 Quittner AL, Buu A, Messer MA, et al. Development and validation of The Cystic Fibrosis Questionnaire in the United States: a health-related quality-of-life measure for cystic fibrosis. Chest 2005; 128: 2347-2354.

16 Dobbin CJ, Bartlett D, Melehan K, et al. The effect of infective exacerbations on sleep and neurobehavioral function in cystic fibrosis. Am J Respir Crit Care Med 2005; 172: 99-104.

17 Oermann CM, Retsch-Bogart GZ Quittner AL, et al. An 18-month study of the safety and efficacy of repeated courses of inhaled aztreonam lysine in cystic fibrosis. Pediatr Pulmonol 2010; 45: 1121-1134.

18 Tiddens HA. Detecting early structural lung damage in cystic fibrosis. Pediatr Pulmonol 2002; 34: 228-231.

19 Que C, Cullinan P, Geddes D. Improving rate of decline of FEV1 in young adults with cystic fibrosis. Thorax 2006; 61: 155-157.

20 Gibson RL, Burns JL, Ramsey BW. Pathophysiology and management of pulmonary infections in cystic fibrosis. Am J Respir Crit Care Med 2003; 168: 918-951.

21 Bedrossian CW, Greenberg SD, Singer DB, et al. The lung in cystic fibrosis. A quantitative study including prevalence of pathologic findings among different age groups. Hum Pathol 1976; 7: 195-204.

22 Tiddens HA, Pare PD, Hogg JC, et al. Cartilaginous airway dimensions and airflow obstruction in human lungs. Am J Respir Crit Care Med 1995; 152: 260-266.

23 Tiddens HA, Koopman LP, Lambert RK, et al. Cartilaginous airway wall dimensions and airway resistance in cystic fibrosis lungs. Eur Respir J 2000; 15: 735-742.

24 Cosio M, Ghezzo H, Hogg JC, et al. The relations between structural changes in small airways and pulmonaryfunction tests. N Engl J Med 1978; 298: 1277-1281.

25 Leigh MW, Kylander JE, Yankaskas JR, et al. Cell proliferation in bronchial epithelium and submucosal glands of cystic fibrosis patients. Am J Respir Cell Mol Biol 1995; 12: 605-612.

26 Dovey M, Wisseman CL, Roggli VL, et al. Ultrastructural morphology of the lung in cystic fibrosis. J Submicrosc Cytol Pathol 1989; 21: 521-534.

27 Kang, EY, Miller RR, Muller NL. Bronchiectasis: comparison of preoperative thin-section CT and pathologic findings in resected specimens. Radiology 1995; 195: 649-654.

28 de Jong PA, Ottink MD, Robben SG, et al. Pulmonary disease assessment in cystic fibrosis: comparison of CT scoring systems and value of bronchial and arterial dimension measurements. Radiology 2004; 231: 434-439.

29 Loeve M, van Hal PT, Robinson P, et al. The spectrum of structural abnormalities on CT scans from patients with CF with severe advanced lung disease. Thorax 2009; 64: 876-882.

30 Mumcuoglu EU, Prescott J, Baker BN, et al. Image analysis for cystic fibrosis: automatic lung airway wall and vessel measurement on CT images. Conf Proc IEEE Eng Med Biol Soc 2009; 2009: 3545-3548.

31 Montaudon M, Berger P, Cangini-Sacher A, et al. Bronchial measurement with three-dimensional quantitative thin-section CT in patients with cystic fibrosis. Radiology 2007; 242: 573-581.

32 Goris ML, Zhu HJ, Blankenberg F, et al. An automated approach to quantitative air trapping measurements in mild cystic fibrosis. Chest 2003; 123: 1655-1663.

33 Bonnel AS, Song SM, Kesavarju K, et al. Quantitative air-trapping analysis in children with mild cystic fibrosis lung disease. Pediatr Pulmonol 2004; 38: 396-405.

34 Loeve M, de Bruijne M, Gorbunova V, et al. Reversibility of trapped air on CT scans of cystic fibrosis patients, an automated approach. J Cystic Fibrosis 2011; 10: S51.

35 Robinson TE, Goris ML, Zhu HJ, et al. Dornase alfa reduces air trapping in children with mild cystic fibrosis lung disease: a quantitative analysis. Chest 2005; 128: 2327-2335.

36 Odry BL, Kiraly AP, Godoy MC, et al. Automated CT scoring of airway diseases: preliminary results. Acad Radiol 2010; 17: 1136-1145.

37 Ross JC, San Jose Estepar R, Kindlmann G, et al. Automatic lung lobe segmentation using particles, thin plate splines, and maximum a posteriori estimation. Med Image Comput Comput Assist Interv 2010; 13: $163-171$.

38 Petersen J, Nielsen M, Lo P, et al. Optimal graph based segmentation using flow lines with application to airway wall segmentation. Inf Process Med Imaging 2011; 22: 49-60.

39 Weinheimer O, Achenbach T, Bletz C, et al. About objective 3-d analysis of airway geometry in computerized tomography. IEEE Trans Med Imaging 2008; 27: 64-74.

40 Tschirren J, Hoffman EA, McLennan G, et al. Segmentation and quantitative analysis of intrathoracic airway trees from computed tomography images. Proc Am Thorac Soc 2005; 2, 484-7: 503-504.

41 Tschirren J, McLennan G, Palagyi K, et al. Matching and anatomical labeling of human airway tree. IEEE Trans Med Imaging 2005; 24: 1540-1547.

42 Palagyi K, Tschirren J, Hoffman EA, et al. Quantitative analysis of pulmonary airway tree structures. Comput Biol Med 2006; 36: 974-996.

43 Brillet PY, Fetita CI, Beigelman-Aubry C, et al. Grenier, Quantification of bronchial dimensions at MDCT using dedicated software. Eur Radiol 2007; 17: 1483-1489.

44 Fetita CI, Preteux F, Beigelman-Aubry C, et al. Pulmonary airways: 3-D reconstruction from multislice CT and clinical investigation. IEEE Trans Med Imaging 2004; 23: 1353-1364. 
Goris ML, Zhu HJ, Robinson TE. A critical discussion of computer analysis in medical imaging. Proc Am Thorac Soc 2007; 4: 347-349.

46 Hansell DM. Bronchiectasis. Radiol Clin North Am 1998; 36: 107-128.

47 Stick SM, Brennan S, Murray C, et al. Bronchiectasis in infants and preschool children diagnosed with cystic fibrosis after newborn screening. J Pediatr 2009; 155: 623-628.

48 Long FR. High-resolution computed tomography of the lung in children with cystic fibrosis: technical factors. Proc Am Thorac Soc 2007; 4: 306-309.

49 Martinez TM, Llapur CJ, Williams TH, et al. High-resolution computed tomography imaging of airway disease in infants with cystic fibrosis. Am I Respir Crit Care Med 2005; 172: 1133-1138.

50 Sly PD, Brennan S, Gangell C, et al. Lung disease at diagnosis in infants with cystic fibrosis detected by newborn screening. Am J Respir Crit Care Med 2009; 180: 146-152.

51 de Jong PA, Nakano Y, Lequin MH, et al. Progressive damage on high resolution computed tomography despite stable lung function in cystic fibrosis. Eur Respir J 2004; 23: 93-97.

52 de Jong PA, Lindblad A, Rubin L, et al. Progression of lung disease on computed tomography and pulmonary function tests in children and adults with cystic fibrosis. Thorax 2006; 61: 80-85.

53 Mott LS, Park J, Murray CP, et al. Progression of early structural lung disease in young children with cystic fibrosis assessed using CT. Thorax 2012; 67: 509-516.

54 Wainwright CE, Vidmar S, Armstrong DS, et al. Effect of bronchoalveolar lavage-directed therapy on Pseudomonas aeruginosa infection and structural lung injury in children with cystic fibrosis: a randomized trial. Jama 2011; 306: $163-171$.

55 Shah RM, Sexauer W, Ostrum BJ, et al. High-resolution CT in the acute exacerbation of cystic fibrosis: evaluation of acute findings, reversibility of those findings, and clinical correlation. AJR Am J Roentgenol 1997; 169: 375-380.

56 Robinson TE, Leung AN, Northway WH, et al. Spirometer-triggered high-resolution computed tomography and pulmonary function measurements during an acute exacerbation in patients with cystic fibrosis. J Pediatr 2001; 138: 553-559.

57 Brody AS, Molina PL, Klein JS, et al. High-resolution computed tomography of the chest in children with cystic fibrosis: support for use as an outcome surrogate. Pediatr Radiol 1999; 29: 731-735.

58 Davis SD, Fordham LA, Brody AS, et al. Computed tomography reflects lower airway inflammation and tracks changes in early cystic fibrosis. Am J Respir Crit Care Med 2007; 175: 943-950.

59 Robinson TE, Leung AN, Northway WH, et al. Composite spirometric-computed tomography outcome measure in early cystic fibrosis lung disease. Am J Respir Crit Care Med 2003; 168: 588-593.

60 Nasr SZ, Kuhns LR, Brown RW, et al. Use of computerized tomography and chest x-rays in evaluating efficacy of aerosolized recombinant human DNase in cystic fibrosis patients younger than age 5 years: a preliminary study. Pediatr Pulmonol 2001; 31: 377-382.

61 Nasr SZ, Gordon D, Sakmar E, et al. High resolution computerized tomography of the chest and pulmonary function testing in evaluating the effect of tobramycin solution for inhalation in cystic fibrosis patients. Pediatr Pulmonol 2006; 41: 1129-1137.

62 Nasr SZ, Sakmar E, Christodoulou E, et al. The use of high resolution computerized tomography (HRCT) of the chest in evaluating the effect of tobramycin solution for inhalation in cystic fibrosis lung disease. Pediatr Pulmonol 2010; 45: 440-449.

63 de Jong PA, Long FR, Nakano Y. Computed tomography dose and variability of airway dimension measurements: how low can we go? Pediatr Radiol 2006; 36: 1043-1047.

64 Loeve M, Lequin MH, de Bruijne M, et al. Cystic fibrosis: are volumetric ultra-low-dose expiratory CT scans sufficient for monitoring related lung disease? Radiology 2009; 253: 223-229.

65 Robinson TE, Leung AN, Moss RB, et al. Standardized high-resolution CT of the lung using a spirometer-triggered electron beam CT scanner. AJR Am J Roentgenol 1999; 172: 1636-168.

66 Robinson TE. Computed tomography scanning techniques for the evaluation of cystic fibrosis lung disease. Proc Am Thorac Soc 2007; 4: 310-315.

67 Mueller KS, Long FR, Flucke RL, et al. Volume-monitored chest CT: a simplified method for obtaining motion-free images near full inspiratory and end expiratory lung volumes. Pediatr Radiol 2010; 40: 1663-1669.

68 Kohz P, Stabler A, Beinert T, et al. Reproducibility of quantitative, spirometrically controlled CT. Radiology 1995; 197: 539-542.

69 Long FR, Williams RS, Adler BH, et al. Comparison of quiet breathing and controlled ventilation in the highresolution CT assessment of airway disease in infants with cystic fibrosis. Pediatr Radiol 2005; 35: 1075-1080.

70 Tiddens HA, de Jong PA. Imaging and clinical trials in cystic fibrosis. Proc Am Thorac Soc 2007; 4: 343-346.

71 Robinson TE, Leung AN, Chen X, et al. Cystic fibrosis HRCT scores correlate strongly with Pseudomonas infection. Pediatr Pulmonol 2009; 44: 1107-1117.

72 Pillarisetti N, Williamson E, Linnane B, et al. Infection, inflammation, and lung function decline in infants with cystic fibrosis. Am J Respir Crit Care Med 2011; 184: 75-81.

73 Carlsson M, Shukla S, Petersson AC, et al. Pseudomonas aeruginosa in cystic fibrosis: pyocyanin negative strains are associated with BPI-ANCA and progressive lung disease. J Cyst Fibros 2011; 10: 265-271.

74 Brody AS, Sucharew H, Campbell JD, et al. Computed tomography correlates with pulmonary exacerbations in children with cystic fibrosis. Am J Respir Crit Care Med 2005; 172: 1128-1132.

75 Loeve M, Gerbrands K, Hop WC, et al. Bronchiectasis and pulmonary exacerbations in children and young adults with cystic fibrosis. Chest 2011; 140: 178-185.

76 Tepper LA, Utens EM, Quittner AL, et al. Impact of bronchiectasis on quality of life in cystic fibrosis lung disease. Pediatric Pulmonology 2010; 373: abstract 428 .

77 Loeve M, Hop WC, de Bruijne M, et al. Chest computed tomography scores are predictive of survival in patients with cystic fibrosis awaiting lung transplantation. Am J Respir Crit Care Med 2012; 185: 1096-1103.

78 Liou TG, Adler FR, Huang D. Use of lung transplantation survival models to refine patient selection in cystic fibrosis. Am J Respir Crit Care Med 2005; 171: 1053-1059.

79 Mayer-Hamblett N, Rosenfeld M, Emerson J, et al. Developing cystic fibrosis lung transplant referral criteria using predictors of 2-year mortality. Am J Respir Crit Care Med 2002; 166: 1550-1555. 
80 Vizza CD, Yusen RD, Lynch JP, et al. Outcome of patients with cystic fibrosis awaiting lung transplantation. Am J Respir Crit Care Med 2000; 162: 819-825.

81 Belkin RA, Henig NR, Singer LG, et al. Risk factors for death of patients with cystic fibrosis awaiting lung transplantation. Am J Respir Crit Care Med 2006; 173: 659-666.

82 Egan TM, Bennett LE, Garrity ER, et al. Predictors of death on the UNOS lung transplant waiting list: results of a multivariate analysis. J Heart Lung Transplant 2001; 20: 242.

83 Kerem E, Reisman J, Corey M, et al. Prediction of mortality in patients with cystic fibrosis. N Engl J Med 1992; 326: 1187-1191.

84 Emerson J, Rosenfeld M, McNamara S, et al. Pseudomonas aeruginosa and other predictors of mortality and morbidity in young children with cystic fibrosis. Pediatr Pulmonol 2002; 34: 91-100.

85 Corey M, Farewell V. Determinants of mortality from cystic fibrosis in Canada, 1970-1989. Am J Epidemiol 1996; 143: 1007-1017.

86 Corey M, Edwards L, Levison $\mathrm{H}$, et al. Longitudinal analysis of pulmonary function decline in patients with cystic fibrosis. J Pediatr 1997; 131: 809-814.

87 Schluchter MD, Konstan MW, Drumm ML, et al. Classifying severity of cystic fibrosis lung disease using longitudinal pulmonary function data. Am J Respir Crit Care Med 2006; 174: 780-786.

88 Schluchter MD, Konstan MW, Davis PB. Jointly modelling the relationship between survival and pulmonary function in cystic fibrosis patients. Stat Med 2002; 21: 1271-1287.

89 Sanders DB, Bittner RC, Rosenfeld M, et al. Pulmonary exacerbations are associated with subsequent FEV1 decline in both adults and children with cystic fibrosis. Pediatr Pulmonol 2011; 46: 393-400.

90 Helbich TH, Heinz-Peer G, Eichler I, et al. Cystic fibrosis: CT assessment of lung involvement in children and adults. Radiology 1999; 213: 537-544.

91 Demirkazik FB, Ariyurek OM, Ozcelik U, et al. High resolution CT in children with cystic fibrosis: correlation with pulmonary functions and radiographic scores. Eur J Radiol 2001; 37: 54-59.

92 Amadori A, Antonelli A, Balteri I, et al. Recurrent exacerbations affect FEV(1) decline in adult patients with cystic fibrosis. Respir Med 2009; 103: 407-413.

93 Konstan MW, Wagener JS, VanDevanter DR. Characterizing aggressiveness and predicting future progression of CF lung disease. J Cyst Fibros 2009; 8: Suppl. 1, S15-S19.

94 Jarad NA, Higgs S, Jeffcote T, et al. Factors associated with reduced FEV1 in adult patients with cystic fibrosis in a relatively affluent area. Chron Respir Dis 2005; 2: 133-137.

95 Griese M, Essl R, Schmidt R, et al. Sequential analysis of surfactant, lung function and inflammation in cystic fibrosis patients. Respir Res 2005; 6: 133 .

96 Texereau J, Jamal D, Choukroun G, et al. Determinants of mortality for adults with cystic fibrosis admitted in Intensive Care Unit: a multicenter study. Respir Res 2006; 7: 14.

97 Judge EP, Dodd JD, Masterson JB, et al. Pulmonary abnormalities on high-resolution CT demonstrate more rapid decline than FEV1 in adults with cystic fibrosis. Chest 2006; 130: 1424-1432.

98 Sanders DB, Li Z, Brody AS, et al. Chest CT scores of severity are associated with future lung disease progression in children with CF. Am J Respir Crit Care Med 2011; 184: 816-821.

99 Rosenberg SM, Schramm CM. Predictive value of pulmonary function testing during pulmonary exacerbations in cystic fibrosis. Pediatr Pulmonol 1993; 16: 227-235.

100 Redding GJ, Restuccia R, Cotton EK, et al. Serial changes in pulmonary functions in children hospitalized with cystic fibrosis. Am Rev Respir Dis 1982; 126: 31-36.

101 Bradley JM, Wallace ES, Elborn JS, et al. An audit of the effect of intravenous antibiotic treatment on spirometric measures of pulmonary function in cystic fibrosis. Ir J Med Sci 1999; 168: 25-28.

102 Gozal D, Bailey SL, Keens TG. Evolution of pulmonary function during an acute exacerbation in hospitalized patients with cystic fibrosis. Pediatr Pulmonol 1993; 16: 347-353.

103 Ren CL, Brucker JL, Rovitelli AK, et al. Changes in lung function measured by spirometry and the forced oscillation technique in cystic fibrosis patients undergoing treatment for respiratory tract exacerbation. Pediatr Pulmonol 2006; 41: 345-349.

104 Sanders DB, Hoffman LR, Emerson J, et al. Return of FEV1 after pulmonary exacerbation in children with cystic fibrosis. Pediatr Pulmonol 2010; 45: 127-134.

105 VanDevanter DR, O’Riordan MA, Blumer JL, et al. Assessing time to pulmonary function benefit following antibiotic treatment of acute cystic fibrosis exacerbations. Respir Res 2010; 11: 137.

106 Jaques A, Daviskas E, Turton JA, et al. Inhaled mannitol improves lung function in cystic fibrosis. Chest 2008; 133: $1388-1396$.

107 Ryan G, Mukhopadhyay S, Singh M. Nebulised anti-pseudomonal antibiotics for cystic fibrosis. Cochrane Database Syst Rev 2003; 3: CD001021.

108 Jones AP, Wallis CE. Recombinant human deoxyribonuclease for cystic fibrosis. Cochrane Database Syst Rev 2003; 3: CD001127.

109 Robinson M, Regnis JA, Bailey DL, et al. Effect of hypertonic saline, amiloride, and cough on mucociliary clearance in patients with cystic fibrosis. Am J Respir Crit Care Med 1996; 153: 1503-1059.

110 Saiman L, Marshall BC, Mayer-Hamblett N, et al. Azithromycin in patients with cystic fibrosis chronically infected with Pseudomonas aeruginosa: a randomized controlled trial. Jama 2003; 290: 1749-1756.

111 Eng PA, Morton J, Douglass JA, et al. Short-term efficacy of ultrasonically nebulized hypertonic saline in cystic fibrosis. Pediatr Pulmonol 1996; 21: 77-83.

112 Carr RR, Nahata MC. Azithromycin for improving pulmonary function in cystic fibrosis. Ann Pharmacother 2004; 38: $1520-1524$.

113 Christopher F, Chase D, Stein K, et al. rhDNase therapy for the treatment of cystic fibrosis patients with mild to moderate lung disease. J Clin Pharm Ther 1999; 24: 415-426.

114 Pai VB, Nahata MC. Efficacy and safety of aerosolized tobramycin in cystic fibrosis. Pediatr Pulmonol 2001; 32: 314-327.

115 Nguyen T, Louie SG, Beringer PM, et al. Potential role of macrolide antibiotics in the management of cystic fibrosis lung disease. Curr Opin Pulm Med 2002; 8: 521-528. 
116 Burkhardt O, Lehmann C, Madabushi R, et al. Once-daily tobramycin in cystic fibrosis: better for clinical outcome than thrice-daily tobramycin but more resistance development? J Antimicrob Chemother 2006; 58: 822-829.

117 Chuchalin A, Csiszer E, Gyurkovics K, et al. A formulation of aerosolized tobramycin (Bramitob) in the treatment of patients with cystic fibrosis and Pseudomonas aeruginosa infection: a double-blind, placebo-controlled, multicenter study. Paediatr Drugs 2007; 9: Suppl. 1, 21-31.

118 Latzin P, Fehling M, Bauernfeind A, et al. Efficacy and safety of intravenous meropenem and tobramycin versus ceftazidime and tobramycin in cystic fibrosis. J Cyst Fibros 2008; 7: 142-146.

119 McCoy KS, Quittner AL, Oermann CM, et al. Inhaled aztreonam lysine for chronic airway Pseudomonas aeruginosa in cystic fibrosis. Am J Respir Crit Care Med 2008; 178: 921-928.

120 Konstan MW, Flume PA, Kappler M, et al. Safety, efficacy and convenience of tobramycin inhalation powder in cystic fibrosis patients: The EAGER trial. J Cyst Fibros 2011; 10: 54-61.

121 Cooper PJ, Robertson CF, Hudson IL, et al. Variability of pulmonary function tests in cystic fibrosis. Pediatr Pulmonol 1990; 8: 16-22.

122 Chavasse R, Johnson P, Francis J, et al. To clip or not to clip? Noseclips for spirometry. Eur Respir J 2003; 21: 876-878.

123 Sanders DB, Rosenfeld M, Mayer-Hamblett N. D. Stamey, and G.J. Redding, Reproducibility of spirometry during cystic fibrosis pulmonary exacerbations. Pediatr Pulmonol 2008; 43: 1142-1146.

124 Nickerson BG, Lemen RJ, Gerdes CB, et al. Within-subject variability and per cent change for significance of spirometry in normal subjects and in patients with cystic fibrosis. Am Rev Respir Dis 1980; 122: 859-866.

125 Hutchison AA, Erben A, McLennan LA, et al. Intrasubject variability of pulmonary function testing in healthy children. Thorax 1981; 36: 370-377.

126 Stanbrook MB, Corey M, Tullis DE. The repeatability of forced expiratory volume measurements in adults with cystic fibrosis. Chest 2004; 125: 150-155.

127 Kangalee KM, Abboud RT. Interlaboratory and intralaboratory variability in pulmonary function testing. A 13-year study using a normal biologic control. Chest 1992; 101: 88-92.

128 Loeb JS, Blower WC, Feldstein JF, et al. Acceptability and repeatability of spirometry in children using updated ATS/ERS criteria. Pediatr Pulmonol 2008; 43: 1020-1024.

129 Gaffin JM, Shotola NL, Martin TR, et al. Clinically useful spirometry in preschool-aged children: evaluation of the 2007 American Thoracic Society Guidelines. J Asthma 2010; 47: 762-767.

130 Stenbit AE, Flume PA. Pulmonary exacerbations in cystic fibrosis. Curr Opin Pulm Med 2011; 17: 442-447.

131 Sawicki GS, Rasouliyan L, McMullen AH, et al. Longitudinal assessment of health-related quality of life in an observational cohort of patients with cystic fibrosis. Pediatr Pulmonol 2011; 46: 36-44.

132 Tluczek A, Becker T, Laxova A, et al. Relationships among health-related quality of life, pulmonary health, and newborn screening for cystic fibrosis. Chest 2011; 140: 170-177.

133 Havermans T, Colpaert K, Vanharen L, et al. Health related quality of life in cystic fibrosis: To work or not to work? J Cyst Fibros 2009; 8: 218-223.

134 Wahl AK, Rustoen T, Hanestad BR, et al. Living with cystic fibrosis: impact on global quality of life. Heart Lung 2005; 34: 324-331.

135 Thomas C, Mitchell P, O'Rourke P, et al. Quality-of-life in children and adolescents with cystic fibrosis managed in both regional outreach and cystic fibrosis center settings in Queensland. J Pediatr 2006; 148: 508-516.

136 Friedman EM, Stewart M. An assessment of sinus quality of life and pulmonary function in children with cystic fibrosis. Am I Rhinol 2006; 20: 568-572.

137 Riekert KA, Bartlett SJ, Boyle MP, et al. The association between depression, lung function, and health-related quality of life among adults with cystic fibrosis. Chest 2007; 132: 231-237.

138 Bregnballe V, Thastum M, Lund LD, et al. Validation of the Danish version of the revised cystic fibrosis quality of life questionnaire in adolescents and adults (CFQ-R14+). J Cyst Fibros 2008; 7: 531-536.

139 Klijn PH, van Stel HF, Quittner AL, et al. Validation of the Dutch cystic fibrosis questionnaire (CFQ) in adolescents and adults. J Cyst Fibros 2004; 3: 29-36.

140 Gee L, Abbott J, Hart A, et al. Associations between clinical variables and quality of life in adults with cystic fibrosis. J Cyst Fibros 2005; 4: 59-66.

141 Bradley J, Dempster M, Wallace E, et al. The adaptations of a quality of life questionnaire for routine use in clinical practice: the Chronic Respiratory Disease Questionnaire in cystic fibrosis. Qual Life Res 1999; 8: 65-71.

142 Powers PM, Gerstle R, Lapey A. Adolescents with cystic fibrosis: family reports of adolescent health-related quality of life and forced expiratory volume in one second. Pediatrics 2001; 107: E70.

143 Orenstein DM, Nixon PA, Ross EA, et al. Kaplan, The quality of well-being in cystic fibrosis. Chest 1989; 95: 344-347.

144 Courtney JM, Kelly MG, Watt A, et al. Quality of life and inflammation in exacerbations of bronchiectasis. Chron Respir Dis 2008; 5: 161-168.

145 Gustafsson PM, De Jong PA, Tiddens HA, et al. Multiple-breath inert gas washout and spirometry versus structural lung disease in cystic fibrosis. Thorax 2008; 63: 129-134.

146 Ellemunter H, Fuchs SI, Unsinn KM, et al. Sensitivity of Lung Clearance Index and chest computed tomography in early CF lung disease. Respir Med 2010; 104: 1834-1842.

147 Owens CM, Aurora P, Stanojevic S, et al. Lung Clearance Index and HRCT are complementary markers of lung abnormalities in young children with CF. Thorax 2011; 66: 481-488.

148 Aurora P, Gustafsson P, Bush A, et al. Multiple breath inert gas washout as a measure of ventilation distribution in children with cystic fibrosis. Thorax 2004; 59: 1068-1073.

149 Aurora P, Bush A, Gustafsson P, et al. Multiple-breath washout as a marker of lung disease in preschool children with cystic fibrosis. Am J Respir Crit Care Med 2005; 171: 249-256.

150 Gustafsson PM, Aurora P, Lindblad A. Evaluation of ventilation maldistribution as an early indicator of lung disease in children with cystic fibrosis. Eur Respir J 2003; 22: 972-979.

151 Horsley AR, Gustafsson PM, Macleod KA, et al. Lung clearance index is a sensitive, repeatable and practical measure of airways disease in adults with cystic fibrosis. Thorax 2008; 63: 135-140.

152 Lum S, Gustafsson P, Ljungberg H, et al. Early detection of cystic fibrosis lung disease: multiple-breath washout versus raised volume tests. Thorax 2007; 62: 341-347.

153 de Jong PA, Mayo JR, Golmohammadi K, et al. Estimation of cancer mortality associated with repetitive computed tomography scanning. Am J Respir Crit Care Med 2006; 173: 199-203. 
154 Pearce MS, Salotti JA, Little MP, et al. Radiation exposure from CT scans in childhood and subsequent risk of leukaemia and brain tumours: a retrospective cohort study. Lancet 2012; 380: 499-505.

155 O'Connor OJ, Vandeleur M, McGarrigle AM, et al. Development of low-dose protocols for thin-section CT assessment of cystic fibrosis in pediatric patients. Radiology 2010; 257: 820-829.

156 Brody AS, Frush DP, Huda W, et al. Radiation risk to children from computed tomography. Pediatrics 2007; 120: $677-682$.

157 Egelhoff JC, Ball WS Jr, Koch BL, et al. Safety and efficacy of sedation in children using a structured sedation program. AJR Am J Roentgenol 1997; 168: 1259-1262.

158 Sanborn PA, Michna E, Zurakowski D, et al. Adverse cardiovascular and respiratory events during sedation of pediatric patients for imaging examinations. Radiology 2005; 237: 288-294.

159 Jevtovic-Todorovic V. Developmental synaptogenesis and general anesthesia: a kiss of death? Curr Pharm Des 2012; 18: 6225-6231.

160 McCann ME, Soriano SG. General anesthetics in pediatric anesthesia: influences on the developing brain. Curr Drug Targets 2012; 13: 944-951.

161 Vutskits L. Anesthetic-related neurotoxicity and the developing brain: shall we change practice? Paediatr Drugs 2012; 14: 13-21.

162 American Academy of P, American Academy of Pediatric D, Cote CJ, et al. Guidelines for monitoring and management of pediatric patients during and after sedation for diagnostic and therapeutic procedures: an update. Pediatrics 2006; 118: 2587-2602.

163 Sury M, Bullock I, Rabar S, et al. Sedation for diagnostic and therapeutic procedures in children and young people: summary of NICE guidance. Bmj 2010; 341: c6819.

164 Lell MM, May M, Deak P, et al. High-pitch spiral computed tomography: effect on image quality and radiation dose in pediatric chest computed tomography. Invest Radiol 2011; 46: 116-123.

165 Moss RB, Rodman D, Spencer LT, et al. Repeated adeno-associated virus serotype 2 aerosol-mediated cystic fibrosis transmembrane regulator gene transfer to the lungs of patients with cystic fibrosis: a multicenter, double-blind, placebo-controlled trial. Chest 2004; 125: 509-521.

166 Brody AS. Computed tomography scanning in cystic fibrosis research trials: practical lessons from three clinical trials in the United States. Proc Am Thorac Soc 2007; 4: 350-354. 